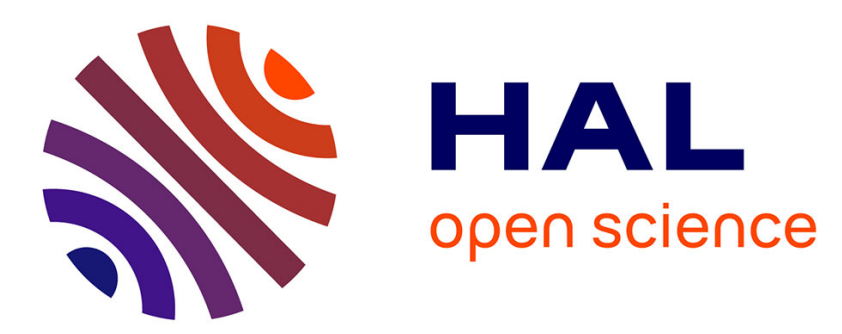

\title{
Laos. Fouilles et prospections dans la région de Vat Phu (province de Champassak, sud du Laos)
}

Marielle Santoni, Christine Hawixbrock

\section{To cite this version:}

Marielle Santoni, Christine Hawixbrock. Laos. Fouilles et prospections dans la région de Vat Phu (province de Champassak, sud du Laos). Bulletin de l'Ecole française d'Extrême-Orient, 1998, 85, pp.387 - 405. 10.3406/befeo.1998.2560 . halshs-02549124

\section{HAL Id: halshs-02549124 \\ https://shs.hal.science/halshs-02549124}

Submitted on 21 Apr 2020

HAL is a multi-disciplinary open access archive for the deposit and dissemination of scientific research documents, whether they are published or not. The documents may come from teaching and research institutions in France or abroad, or from public or private research centers.
L'archive ouverte pluridisciplinaire HAL, est destinée au dépôt et à la diffusion de documents scientifiques de niveau recherche, publiés ou non, émanant des établissements d'enseignement et de recherche français ou étrangers, des laboratoires publics ou privés. 


\section{Laos. Fouilles et prospections dans la région de Vat Phu} (province de Champassak, sud du Laos)

Marielle Santoni, Christine Hawixbrock

\section{Citer ce document / Cite this document :}

Santoni Marielle, Hawixbrock Christine. Laos. Fouilles et prospections dans la région de Vat Phu (province de Champassak, sud du Laos). In: Bulletin de l'Ecole française d'Extrême-Orient. Tome 85, 1998. pp. 387-405; doi : https://doi.org/10.3406/befeo.1998.2560 https://www.persee.fr/doc/befeo_0336-1519_1998_num_85_1_2560

Fichier pdf généré le 08/11/2019 


\section{LAOS}

\section{Fouilles et prospections dans la région de Vat Phu (Province de Champassak, Sud du Laos)}

L'ensemble de Vat Phu (fig. 1) se trouve sur la rive droite du Mékong à environ $30 \mathrm{~km}$ au sud de Paksé et $100 \mathrm{~km}$ au nord des chutes de Khon qui marquent la frontière avec le Cambodge. Il se compose essentiellement d'un complexe monumental religieux (hindouiste) de style khmer classique (Parmentier, 1914) et d'une grande ville à double enceinte, de période préangkorienne (VIe-VIIe siècles). L'ensemble religieux est situé au pied d'une montagne dont le point culminant, le Phu Kao, attire particulièrement l'attention par sa forme, identifiée dans l'antiquité à un linga, d'où son nom ancien de Lingapārvata ${ }^{1}$ et sa réputation de montagne sacrée. La présence d'une source permanente, au pied d'une des falaises, a vraisemblablement incité les anciens rois de la région à installer là un sanctuaire shivaïte. Dans la plaine, à $5 \mathrm{~km}$ à l'est, au bord du Mékong, se trouve la ville préangkorienne dont les vestiges (enceintes de terre crue, nombreux monuments de briques) sont actuellement à peine visibles au sol, quoiqu'ils apparaissent très bien sur les photographies aériennes qui montrent aussi une occupation ancienne très dense de toute la plaine : irrigation et parcellaire anciens, voies de communication, enceintes, monuments, villages. La chaussée ancienne qui relie Vat Phu à la région d'Angkor est particulièrement reconnaissable ; elle est repérable sur presque tout son parcours long de plus de $100 \mathrm{~km}$, et son tracé avait déjà été en partie reporté sur les cartes d'Indochine des années 1950.

Depuis 1991, le P.R.A.L. (Projet de recherches en archéologie lao) ${ }^{2}$ travaille dans la région de Vat Phu ${ }^{3}$. Dans cette chronique seront présentés ses travaux les plus récents (fouille du monument de Nong Vienne ; étude du site de Khan Mak Houk, un rocher sculpté dans le Mékong ; prospections dans le district de Mounlapamok, cf. Hawixbrock, 1998 ; Santoni, 1998), avec seulement un bref rappel des résultats antérieurs.

Les fouilles menées de 1991 à 1995 sur le complexe religieux lui-même, derrière le sanctuaire, ont permis notamment de mettre en évidence un vaste ensemble de captage et d'adduction des eaux de la source sacrée (au pied de la falaise) vers le sanctuaire, pour un ondoiement permanent du linga ${ }^{4}$, ainsi que de confirmer l'existence pressentie d'un temple préangkorien, remplacé par le sanctuaire du XIe siecle.

De 1991 à 1998 ont également eu lieu des prospections et des fouilles dans la ville ancienne. La ville, rectangulaire, mesure environ $2,3 \mathrm{~km} \times 1,8 \mathrm{~km}$. Elle est entourée sur deux côtés (sud et ouest) d'une double enceinte (sorte de douve entre deux levées de terre), le

1. Au sujet du Lingapārvata, voir Cœedès 1989, p. 126.

2. Il s'agit d'un projet de coopération lao-français en archéologie, entre la direction de l'Archéologie et des Musées de la R.D.P. Lao, ministère de l'Information et de la Culture, Vientiane, et le musée national des Arts asiatiques-Guimet, Paris. Le P.R.A.L., codirigé par Viengkèo Souksavatdy et Marielle Santoni, a déjà accompli sept saisons de fouilles et prospections, sur un financement du ministère des Affaires étrangères français.

3. Après quelques tentatives inabouties de 1986 à 1990, l'UNESCO a finalement, en 1997, mis sur pied un programme d'étude et de protection du site en vue de son inscription à l'Inventaire du Patrimoine mondial. Pierre Pichard (EFEO) est chargé d'élaborer un projet de restauration.

4. Voir à ce sujet : Santoni, Defente, Hawixbrock, Liger, Souksavatdy, et Zolese, 1991 ; Santoni, Souksavatdy, Defente, Hawixbrock, et Liger, 1992. 
troisième côté (nord) étant probablement formé par le Houay Sa Houa, une rivière autrefois partiellement canalisée, et le quatrième côté (est) par la rive du Mékong. Elle renferme de nombreux vestiges (essentiellement préangkoriens) : murs de subdivision, monuments de brique, avec quelques éléments architecturaux de pierre, socles de statues, parfois inscrits, et l'on peut trouver en surface des fragments de sculptures, divers outils de pierre (égrugeoirs, pilons, haches), et de la céramique. L'examen et le relevé des structures ont permis de recenser plus de trente monuments religieux, construits en briques et grès, comportant le plus souvent un bassin à l'est. Des zones artisanales, notamment des aires de cuisson de briques, ont été définies, et l'une d'entre elles fouillée (Souksavatdy, 1994).

La ville a pu être fondée vers le milieu du Ve siècle par le roi Devānīka (d'après l'inscription sur la stèle dite « de Vat Luang Kau », trouvée sur place : Cœdès, 1956, Jacques, 1962 et 1986), peut-être sur un village préexistant. Parfois identifiée avec la ville khmère Srestapura, elle fut certainement un grand centre politique aux VIe-VIIe siècles.

L'importance de Vat Phu et de la région était telle que durant tout l'empire khmer, les souverains n'ont cessé d'y faire des fondations, se légitimant en quelque sorte, en se rattachant à la dynastie de Bhavavarman et Mahendravarman, dont nos fouilles confirment qu'elle est issue de cette région. En effet, deux inscriptions de Mahendravarman (Jacques 1993) dédiant des effigies de Nandin à son oncle et à son père ont été trouvées associées à un des monuments de la ville ancienne. Celui-ci, dégagé au cours des campagnes 1993, 1995 et 1996, s'est révélé être un sanctuaire de briques à plan carré avec grand avant-corps, de style très simple, avec marche en demi-lune, qui, si l'on se fie aux catégories définies par Henri Parmentier (Parmentier, 1927 et 1933), pourrait bien dater de la période qui a vu la fin du Funan.

\section{Fouille de Nong Vienne}

En 1996 et 1998, nous avons fouille sur le site de Nong Vienne' (Santoni, 1998 ; Souksavatdy, 1996), au centre de la ville, une structure circulaire qui avait, dès l'examen des photographies aériennes, attiré notre attention. Le site se présentait comme un monticule subrectangulaire (ovale) entouré de douves. L'aspect arrondi des douves évoquait une structure ronde - peut-être un stüpa, pensions-nous - mais cela entrait en contradiction avec l'allongement du monticule. Les investigations de surface n'avaient livré que peu d'informations : à peine quelques briques visibles dans les buissons, aucun tesson ni élément lapidaire.

Cette situation particulière avait donc fait sélectionner ce site pour un examen magnétique. Les mesures, effectuées en 1995, sur une ligne nord-sud, ont montré de fortes anomalies magnétiques. Mauro Cucarzi (Fondation Lerici, Rome) a estimé que l'on était en présence de deux murs au moins, et que l'épaisseur (la hauteur) de la structure devait atteindre environ trois mètres (Cucarzi, Zolese, 1994). Le premier sondage a partiellement confirmé cette interprétation, mais, bien au-delà, nous a livré un résultat assez inattendu.

Après débroussaillage, le site se présentait comme un monticule assez allongé $(55 \mathrm{~m} \mathrm{x}$ $30 \mathrm{~m}$ ) orienté nord-sud, un peu étréci en son milieu $(20 \mathrm{~m})$, s'élevant sur une plate-forme de terre très propre, couverte d'herbe rase, de $110 \mathrm{~m}$ sur $85 \mathrm{~m}$. Les douves, de $22 \mathrm{~m}$ de large en moyenne, portent les mesures de l'ensemble de la structure (douves comprises) à $150 \mathrm{~m}$ de long sur $125 \mathrm{~m}$ de large (fig. 2). Les bords intérieurs portent encore les traces d'aménagement en pierres plates, trait commun à presque toutes les pièces d'eau associées aux monuments dans la ville.

1. Ont participé à cette fouille : Bérénice Bellina, Michel Jacq-Helgouac'h, Jean-Claude Liger, Marielle Santoni, Viengkèo Souksavatdy, Bounlap Kéokangna, Thongkhoun Boliboun, Phimmaseng Thammavong, Bounheuang Bouasisengpraseuth, Sengphet Nokhamsomphou, Tha Sirichantho. 
En 1996, une tranchée de $58 \mathrm{~m}$ de long sur $2 \mathrm{~m}$ de large a été implantée sur un axe nordsud correspondant à celui utilisé pour les mesures magnétiques. Les traces d'une occupation récente (1970) apparaissaient encore en surface. Au premier décapage, des traces évidentes de pillage sont également apparues, sous forme de nappes de galets indiquant des rejets de creusements dans les fondations de la structure. Les habitants du village voisin nous ont finalement confirmé que dans les années 1970, un « archéologue » engagé par le prince Bounhoum $\mathrm{Na}$ Champassak avait creusé une tranchée transversale est-ouest. Mais le site a aussi été dévasté par une grande quantité de vastes et profondes fosses de pillage, plus anciennes. Malgré ces perturbations et l'étroitesse de notre sondage, les vestiges découverts ont permis de donner une première interprétation, entièrement confirmée par les fouilles de 1998, qui ont affecté plus du quart de la superficie du monticule.

Il s'agit de deux structures rondes, mesurant chacune environ $25 \mathrm{~m}$ de diamètre, juxtaposées sur un axe nord-sud. La structure sud a été appelée monument $n^{\circ} 1$, et la structure nord, monument $n^{\circ} 2$. Très massives, elles sont construites en briques (appariées, disposées à carreau et boutisse), sur des fondations de galets très profondes au centre (plus de $2,50 \mathrm{~m}$ ). Chaque structure est composée d'une plate-forme de circulation extérieure entourant le centre surélevé de l'édifice. Il paraît difficile d'y voir autre chose que deux stūpa accolés.

\section{Le monument sud $\left(n^{\circ} 1\right)$}

C'est le mieux conservé des deux et il a été fouillé sur sa moitié ouest (photo. 1). La hauteur maximum préservée est de $1,75 \mathrm{~m}$. La plate-forme extérieure mesure en moyenne $1,80 \mathrm{~m}$ de large sur $0,75 \mathrm{~m}$ de haut (10 assises de briques). La première assise de fondation, qui n'était pas visible lors de l'utilisation du bâtiment, déborde sur l'extérieur de la largeur d'une brique. Cette plateforme enserre une seconde plate-forme plus élevée (hauteur conservée : $1 \mathrm{~m}$ ), dont le mur de soutien va en s'étrécissant par paliers (trois moulures formées par les assises en retrait). Le diamètre est de $21,40 \mathrm{~m}$ à la base et $21 \mathrm{~m}$ au sommet. Le bord de cetie plate-forme supérieure devait être arrondi, comme le suggèrent les quelques briques taillées retrouvées.

Il est vraisemblable que cette seconde plate-forme entourait le stūpa proprement dit placé au centre, à moins qu'il n'y ait eu encore des plates-formes intermédiaires. Il n'en reste rien, le monument ayant été totalement pillé à cet endroit. Seul le système de fondation partiellement préservé nous permet d'évoquer la présence d'une structure centrale massive de $8 \mathrm{~m}$ de diametre.

L'accès de la première à la seconde plate-forme se faisait au sud par un escalier, encadré de deux larges ressauts moulurés formant échiffres ; sur chacun était fixé un panneau décoratif de grès représentant une réduction d'édifice $(\mathrm{H}=0,75 ; \mathrm{L}=0,55 ;$ ép. $=0,15$, sans les tenons). Ces panneaux étaient fixés par deux tenons, l'un au dos, l'autre au pied (fig. 3). Un de ces panneaux décoratifs plaqué sur le ressaut, ainsi que les premières moulures et l'amorce du rétrécissement de la base de l'édifice central sont visibles sur la partie la mieux conservée de l'édifice sud, sur le côté gauche de l'entrée (photo. 2). Le côté droit de l'entrée a été détruit et seul demeure le panneau décoratif de pierre : les pilleurs qui ont commencé leur travail par le centre de l'édifice se sont arrêtés juste sur le tenon arrière du panneau, se rendant compte qu'ils atteignaient l'extérieur.

De l'escalier, seule la première marche de briques est conservée, et l'on distingue l'amorce de la deuxième. Il n'y a pas trace de la présence d'une marche de pierre décorative qui aurait pu être posée en avant de la marche de briques, en revanche on peut supposer l'existence d'un placage de pierre, car la brique de la première marche est retaillée pour former un léger arc de cercle concave. Le ressaut formant échiffre est plaqué sur le mur de soutènement de la plate-forme supérieure. Il mesure $0,90 \mathrm{~m}$ de large sur $1 \mathrm{~m}$ de haut et $0,35 \mathrm{~m}$ de profondeur. Les moulures rappellent et soulignent celles du panneau décoratif de pierre en forme de réduction d'édifice (photo. 3 ). 
Côté ouest, les ressauts, autant qu'on puisse en juger par ce qu'il en reste, semblent former un décrochement moins prononcé mais sont eux aussi ornés de deux panneaux décoratifs : on peut encore voir l'espace ménagé dans le mur pour les tenons dorsaux, et dans le sol de la plate-forme pour les tenons de pied (un fragment de tenon cassé est resté en place). Enfin, les $1 / 8$ de cercles étaient marqués par deux petits ressauts ménageant une niche peu profonde.

Côté nord, le monument est entièrement détruit, sauf le rang de briques débordantes qui marque la base de l'édifice : les pilleurs se sont arrêtés sur la surface de galets supérieure des fondations. Un troisième panneau décoratif, à peu près identique aux deux autres, trouvé sur la pente, bien visible, de la fosse de pillage, semble toutefois nous indiquer que l'on avait, au nord, le même dispositif décoratif qu'au sud et à l'ouest. Le côté est, qui d'après les études géophysiques de 1997 serait également totalement détruit, n'a pas été fouillé.

\section{Le monument nord $\left(n^{\circ} 2\right)$}

La structure nord n'a été fouillée que sur le quart sud. Seule la plate-forme de circulation inférieure est conservée, avec quelques lambeaux du mur de soutènement de la plate-forme supérieure. Sur le côté sud de cette plate-forme (dans l'axe nord-sud des deux monuments), se trouvait, marquant l'accès à cet édifice, une marche de pierre du type « en accolade », portant des volutes sur les côtés (photo. 4). Aucun panneau décoratif n'est apparu. Un petit sondage au nord a montré que seule la base de la plate-forme de circulation inférieure était conservée à cet endroit. Les briques composant cet édifice sont d'un module légèrement supérieur à celui de l'édifice sud, disposées de la même façon (paires de briques à carreau et boutisse), avec parfois des variantes type svastika. La plate-forme inférieure ne comporte que sept assises de briques et sa première assise de briques n'est pas débordante comme celle de l'édifice sud.

\section{Extérieur des bâtiments et fondations}

Des briques et des dallettes de pierre ont été empilées à la jonction des deux édifices (distants de $0,70 \mathrm{~m}$ au plus), créant une sorte de passage de plain-pied entre les deux monuments. À leur pied, le sol, dans les espaces triangulaires formés par les courbes tangentes des deux plates-formes, est recouvert d'un dallage de briques grossier et très irrégulier (photo. 4), comme pour consolider et protéger un endroit où, en saison des pluies, l'eau devait ruisseler. On note d'ailleurs que, là où elle n'est pas étayée, la plate-forme a glissé vers l'extérieur.

D'après les sondages dans le monument sud, les fondations de galets des plates-formes sont peu profondes : de $0,50 \mathrm{~m}$ au bord a $1 \mathrm{~m}$ sous le mur de la plate-forme supérieure, creusées en paliers de $0,20 \mathrm{~cm}$ de hauteur et de $1 \mathrm{~m}$ de long. Le centre de l'édifice présente des fondations plus élaborées : sur plus de $2,50 \mathrm{~m}$ de galets mêlés de sable était construite une plate-forme de briques de quelques assises d'epaisseur, recouverte d'une nouvelle couche de galets et de sable, sur laquelle s'élevait la structure centrale. Le cercle intérieur correspondant à cette structure centrale, visible dans l'épaisseur du mur (dans la tranchée de 1996) est de construction particulièrement soignée. C'est ce qui nous permet de restituer un diamètre de $8 \mathrm{~m}$ pour le stūpa central. Il est peu vraisemblable que celui-ci ait été construit en briques pleines, au vu de la faible quantité de briques éboulées retrouvée. Il s'agissait probablement d'un dôme en briques rempli de terre. Les fondations situees sous l'espace compris entre le mur de la plate-forme supérieure et le cercle intérieur n'ont pu être fouillées. Elles seules pourraient peut-être nous donner une idée de l'élévation du bâtiment, qui pouvait comporter plusieurs plates-formes étagées.

Un sondage (1996) à l'extérieur du monument sud (dans l'axe de la tranchée) nous a fourni des informations intéressantes sur la plate-forme de terre d'environ $1 \mathrm{~m}$ d'épaisseur qui soutient les deux édifices. On voit nettement, à la base de la section, le sédiment argileux rouge naturel, couvert d'une couche de terre grise correspondant à une mise en culture et à une exposition à l'air. Ce niveau, qui constitue le sol contemporain de la construction de la plate- 
forme est recouvert par une épaisse couche de sédiment rouge $(0,70 \mathrm{~m})$ rapporté, provenant du creusement dans le sol naturel soit des fondations du monument, soit des douves. Une mince couche contenant de rares fragments de briques représente le niveau d'occupation du monument, couvert par une couche de surface (sédiment gris fin de rizière). Ainsi est bien mis en évidence le système d'érection de la plate-forme, simplement par rejet des terres provenant des creusements, technique également employée pour la constitution des levées de terre qui entourent la ville, et bien connu par la suite dans le monde khmer.

Une coupe pratiquée en 1998 à la jonction des deux monuments montre une différence de niveau peu significative de $5 \mathrm{~cm}$ entre l'assise de fondation du monument nord et celle du monument sud. On retrouve la même succession d'argile grise jaune et rouge. Il existe de légères dissemblances entre les deux monuments (module des briques, nombre d'assises de la plate-forme, absence de première assise débordante). Si l'on tient compte de la petite disparité de niveau, et de l'impression que le monument nord a été creusé alors qu'une mince couche de terre avait déjà eu le temps de se déposer autour du monument sud, alors on peut émettre l'hypothèse que le monument nord a été construit un peu après le monument sud. Toutefois, il est évident que, par la suite, les deux monuments ont fonctionné en même temps.

Les douves ont également fait l'objet d'un sondage en 1998. Larges de $22 \mathrm{~m}$ au total, elles sont, au centre, profondes de $4 \mathrm{~m}$. Elles sont empierrées sur les bord, d'abord en gradins, puis en pente douce, sur une largeur de $6 \mathrm{~m}$ avec un dénivelé de $1,50 \mathrm{~m}$. Puis la pente s'accentue brusquement et plonge de $2,50 \mathrm{~m}$ de plus. Le chenal central, large de $10 \mathrm{~m}$ environ, forme un $\mathrm{U}$ aux branches largement ouvertes.

\section{Datation et interprétation}

Le site est en cours d'étude et une campagne de fouilles supplémentaire serait peut-être nécessaire pour répondre à toutes les questions qui restent posées. En attendant, les panneaux décoratifs en forme de réduction d'édifice (fig. 3 ) pourraient apporter quelques indications pour la datation de nos structures : ils représentent un édifice de type assez simple, à voúte en berceau et arcature décorée de cinq gros points, couronnée par une sorte de pignon qui, habituellement, sur les exemples connus, est en forme de bouton de lotus. Toutefois, ici, l'interprétation n'est pas aisée, et les pignons des trois panneaux retrouvés sont assez différents. Le tympan porte une demi-fleur stylisée, à longs pétales renflés. De tels motifs se retrouvent sur des édifices du début du VIIe siècle à Sambor Prei Kuk, Han Cei, Ampil Rolum, etc. (Cambodge). La couverture est posée sur une corniche débordante. Le corps de l'édifice porte un décor très simple de pilastres appuyés, en haut et en bas sur une moulure débordante. La porte est toute simple, avec trois dés, comme il est d'usage. Le soubassement, à trois étages, est très élevé, et comporte un escalier central à échiffres sans décor. Dans l'ensemble, le décor est très pauvre, et ne présente aucun des feuillages et volutes typiques du style préangkorien classique.

La marche paraît également d'un type légèrement postérieur à celui des marches en demilune, si fréquent dans la ville ancienne, mais moins élaboré que celui de la maturité de la période préangkorienne. Une datation du tout début du VIIe siècle paraît pour le moment assez probable, quoiqu'une date antérieure ne puisse être exclue. À ce monument (ou à une autre des structures rondes repérées lors des prospections géophysiques de 1997) pourrait appartenir la statue de Buddha debout conservée dans les collections de Champassak.

Si l'on sait, tant par les inscriptions que par des sculptures éparses, que bouddhisme et hindouisme ont cohabité en Asie du Sud-Est dès les débuts de l'indianisation, et que, d'après les annales chinoises, le bouddhisme, florissant à l'époque du Funan, semble avoir été supplanté par l'hindouisme lors de l'émergence du Zhenla (Coedès, 1989, Pelliot, 1903), aucun monument bouddhiste de forme ronde, datant du VIIe siècle ou avant, n'avait encore été découvert dans cette région. 
Reste à expliquer la gémellité des structures : deux stūpa ainsi accolés sur une plate-forme pourraient être le produit d'une fondation familiale (époux, parents). Mais que dire des douves, normalement plutôt associées à un édifice hindouiste ? Enfin, on s'attendrait à trouver, sur un stūpa, quatre entrées, une à chaque point cardinal, ou bien une seule entrée, mais à l'est. La seule entrée conservée ici se trouve au sud, et la première impression des fouilleurs est que les ressauts ouest du monument sud correspondent en fait à une «fausse entrée ». Dans l'ensemble, le monument double de Nong Vienne forme un type de structure très étonnante, surtout pour un bouddhisme encore assez proche du modèle indien.

\section{Khan Mak Houk}

Dans le cadre des prospections effectuées par le P.R.A.L. dans la région de Champassak, l'existence d'un rocher sculpté, non loin de la ville ancienne, nous a été signalée par M. Bounlap Kéokangna, conservateur des monuments de Vat Phu. Une première visite du site a été effectuée en 1995 et, en 1998, une étude plus approfondie a été possible. Ce rocher sculpté, répondant au nom de Khan Mak Houk, fait partie d'un groupe de rochers qui affleurent lors des très basses eaux du Mékong à la pointe sud de l'île de Don Deng (fig. 1). Khan Mak Houk est le rocher situé le plus au sud, pratiquement juste en face du débouché de la rivière Houay Tomo, qui donne son nom à un site khmer préangkorien situé sur ses rives. Compte tenu du fait que le lit du Mékong se déplace latéralement vers la rive ouest, ces rochers devaient à l'origine se trouver presque au centre du fleuve alors que ce groupe est beaucoup plus proche, à l'heure actuelle de la rive est et donc de Houay Tomo (fig. 1).

Ce'rocher, de forme allongée (fig. 4), n'est facilement accessible que sur ses faces est et nord-est grâce à la présence de hauts-fonds. En saison des pluies, il est recouvert par plusieurs mètres d'eau et il n'est visible que lors de grosses saisons sèches. À très basses eaux, comme ce fut le cas en 1995, la face est est à sec, découvrant le fond de sable et la face nord-est n'est plus immergée que de $50 \mathrm{~cm}$ environ, tandis que les faces ouest et nord descendent beaucoup plus profond et sont bordées de rapides. Cette disposition particulière est sans doute à l'origine du choix des faces est et nord-est comme support des sculptures, avec la surface supérieure du rocher. Les bas-reliefs qui le recouvrent relèvent de themes brahmaniques comparables en partie à ceux qui ont été sculptés dans le lit de la rivière de Siem Reap, aux Kulen. D'après leur style, les reliefs du rocher peuvent être datés stylistiquement, comme ceux de la première période, aux Kulen, de l'époque du Baphuon.

Le plat du rocher illustre sur une grande partie de sa surface des alignements de linga, sur les faces est et nord-est, les thèmes vishnouites prédominent tels que le sommeil de Viṣnu sur le näga et la naissance de Brahmā. Cette association de thèmes est également présente aux Kulen ${ }^{1}$ ainsi qu'à Koh $\mathrm{Ker}^{2}$ et à l'embouchure d'une rivière situee non loin de Preah Vihear (Bruno Dagens, communication personnelle). De gauche à droite, la face nord-est est sculptée d'un ascète, d'un poisson, d'une tortue montée (?) par un personnage suivi par Viṣnu couché ; la face est est ornée d'un dieu à quatre bras encadré par deux orantes que regarde un éléphant, et d'un personnage monté sur un éléphant encadré par deux dvärapāla.

\section{Face nord-est, description des scènes}

De gauche à droite, apparaît d'abord un petit ascète (reconnaissable à sa coiffure en chignon) agenouillé, vu de profil, face tournée vers le nord. Le buste légèrement penché vers l'avant, il tient dans ses deux mains tendues un récipient en forme de bol. Ce bol se trouve

1. Voir notamment J. Boulbet et B. Dagens, « Les sites archéologiques de la région du Bhnam Kulen (Phnom Kulen) », Arts asiatiques, t. XXVII, 1973.

2. Voir J. Boisselier, Le Cambodge, t. I, Paris, éd. Picard, 1966, p. 288. 
placé à l'aplomb d'une rigole verticale creusée dans le roc (traversant auparavant une partie du plat du rocher parmi les linga) et semble donc recueillir les eaux qui s'en écoulent (fig. 4). Nous avons tout d'abord supposé que ces eaux ainsi recueillies pouvaient être les eaux d'ondoiement des linga. Cependant, s'il s'agissait bien d'elles, cette rigole, qui figurerait alors le bec déverseur, aurait dô être tourné vers le nord et non vers l'est. Il pourrait plutôt s'agir d'une représentation des eaux du Mékong, qui, sanctifiées par la présence des alignements de linga, figurerait une miniature de la Descente du Gange. Vient ensuite le sommeil de Viṣnu sur le näga Ananta (photo. 5). Viṣnu à quatre bras est allongé sur le flanc droit, la main supérieure droite soutenant sa tête, la main inférieure droite allongée le long du corps tenant apparemment le pommeau de la massue. Les deux mains gauches sont peu lisibles. Le visage est perdu, il a été arraché sans doute à une époque relativement récente. Viṣnu porte des pendants d'oreilles en bouton de lotus, un collier gorgerin et des brassards. Compte tenu de l'érosion de la sculpture (que nous n'avons pu voir que sous l'eau), peu de détails sont conservés. Le sampot est par contre bien visible, descendant jusqu'aux genoux, il est lisse et possède une longue chute en ancre frontale apparemment également lisse. Lakșmī (ou Bhumì ?), dont la tête a également été arrachée, est agenouillée à ses pieds, elle soutient sur ses genoux les mollets de Viṣnu, et ses mains semblent reposer sur les jambes du dieu. Les pieds de ce dernier, au lieu d'être dans le prolongement des jambes, sont ramenés vers l'avant en une pliure peu naturaliste. À gauche de la tête de Lakșmì, un petit personnage assis dans une mandorle ovale, peu lisible, pourrait figurer le Soleil ou la Lune. Du nombril de Viṣnu, semble naître Brahmā, puisqu'un personnage complètement disparu, arraché, est situé à l'aplomb du nombril de Vișnu. D'autre part, le départ de la hampe de lotus qui le supporte est en partie visible. Le näga Ananta sur lequel repose le dieu a été sculpté dans un méplat du rocher. Ses écailles et sa queue relevée sont bien visibles à la différence de sa tête, dont les capuchons multiples, qui devraient normalement être redressés au-dessus de Viṣnu, ne peuvent être distingués, sauf peut-être à la base. Il est possible qu'ils ne soient plus visibles car trop érodés à moins qu'ils n'aient jamais été sculptés. Fn effet, à leur niveau, a été figurée une tortue, vue de profil, tête dressée tournée vers le sud. Cependant, contre la tortue, un arrachement vertical dans le grès pourrait indiquer l'emplacement des capuchons du näga. Sous la tortue, dont on distingue bien la partie ventrale et les nageoires, le corps du nāga se prolonge, reconnaissable à ses écailles, à l'endroit où devrait se situer la tête, orné d'un cercle nettement marqué contenu dans un second cercle. En-dessous, on remarque un élément triangulaire se terminant en pointe, couvert d'écailles, et qui appartient peut-être au corps du näga. Il est possible que ce motif, au demeurant difficile à identifier, indique la présence de la tête du nāga qui dans ce cas serait complètement couché. Il est également envisageable que la tortue corresponde à une retaille, bien que ses parures soient du même style que celle des autres divinités.

Du sommet de la carapace de la tortue, émerge le torse d'un personnage masculin au cou orné d'un large collier dont, encore une fois, la tête a été volontairement brisée. Les bras sont ornés de brassards bien visibles mais, compte tenu de l'état du grès, il n'est pas absolument certain que ce personnage possédait quatre bras. Cependant la trace d'un arrachement côté droit, vertical et longiligne pourrait laisser supposer l'existence d'un bras supérieur. À gauche, ce détail n'est pas perceptible et le ou les bras sont brisés sous le brassard. La main inférieure (?) droite est conservée, elle est ramenée contre le buste et tient un attribut ayant la forme d'un «éventail », qui ne semble pas pouvoir être identifié à une massue courte dans la mesure où l'objet n'est pas saisi à pleine main mais semble être tenu entre le pouce et l'index dressés, les autres doigts semblant repliés vers la paume. Il reste donc à préciser la nature de cet objet (inachevé ?) mais il semble d'ores et déjà possible de reconnaître dans ce personnage anthropomorphe une représentation de Viṣnu Kūrma. Il s'agirait dans ce cas de la plus ancienne représentation anthropomorphe de Viṣnu Tortue connue dans un contexte khmer puisque jusqu'à présent ce type iconographique n'était pas antérieur au style du Bayon (fin XIIe siècle) et n'était d'ailleurs connu qu'en ronde bosse. La figuration la plus commune de Viṣnu Kürma, en bas-relief, est celle d'une simple tortue qui n'est normalement figurée, sur les 
frontons et linteaux, que lors du Barattage de l'océan de Lait, puisqu'elle sert de support à la baratte. Or, ce mythe n'est pas illustré ici. Au-dessus de la tête de la tortue, un petit poisson vu de profil, tourné vers le sud, à haute nageoire dorsale, pourrait figurer Vișnu Matsya ou l'un des habitants du milieu aquatique dans lequel est sensé se déplacer Viṣnu Tortue. Un dernier personnage, figuré aux pieds de Vișṇu, ferme en quelque sorte la scène, il s'agit d'un dvärapāla debout qui tient à deux mains, entre ses jambes, une massue courte.

\section{Face est, description des scènes}

Cette face n'est sculptée que de deux thèmes, distincts l'un de l'autre. À gauche, une divinité masculine de petites dimensions, de face, jambes fléchies est figurée à quatre bras (photo. 6). Il s'agit sans doute de Viṣnu puisqu'il porte, en main inférieure droite, une massue courte posée sur l'épaule et, en main supérieure droite, un attribut qui semble correspondre par sa forme au disque ou à la conque. Les deux bras gauches sont dressés vers le haut, les mains se rejoignant pour tenir ensemble une longue guirlande de fleurs ou un serpent. Vișnu, dont le visage est illisible, mais dont on distingue encore les pendants d'oreilles en bouton de lotus, porte un lourd collier en accolade dont les détails sont érodés et un sampot court repassé entre les jambes tandis que le torse reste nu. Deux petites orantes, les jambes repliées dans l'attitude du vol, les mains jointes au-dessus de leur tête, l'encadrent. Côté nord, vient ensuite un éléphant figuré de profil, tête tournée vers l'ouest, c'est-à-dire vers Viṣnu. Sa trompe, dressée, semble tenir soit un lotus, soit un bouquet. Il pourrait également s'agir d'un Viṣnu à quatre bras qui sauve du crocodile le roi Indradyumna réincarné en éléphant. Sous la figure à quatre bras, une forme érodée sous le rocher pourrait faire penser à un crocodile. Cependant Viṣnu devrait alors chevaucher Garuḍa, ce qui ne semble pas être le cas.

Toujours sur la face est, côté nord, un personnage complètement érodé, ressemblant à l'heure actuelle à une "flamme », est assis sur l'encolure d'un éléphant monocéphale vu de face dont seule la tête, d'assez grandes dimensions, a été sculptée. Deux personnages de haute taille, aux visages brisés, tiennent à deux mains le manche d'une massue tenue entre les jambes (photo. 7). Il pourrait dans ce cas s'agir de la figuration d'Indra monté sur Airāvata, encadré par deux dvārapäla.

\section{Le plat du rocher}

La surface supérieure du rocher est sculptée de plusieurs groupes de linga. Sept ensembles d'importance variable sont visibles, mais ils n'existent plus qu'à l'état de trace. En effet, les linga eux-mêmes ont été arrachés par malveillance, comme l'ont été les visages des personnages décrits plus haut, et jetés dans le Mékong. Quatre-vingt-deux linga ont pu être retrouvés sous l'eau autour du rocher. Or, si l'on fait le décompte des traces de linga encore visibles sur le plat du rocher, on arrive à dénombrer, tous groupes confondus, 71 linga, ce qui prouve que le nombre réel était plus élevé et que les traces que nous avons relevées ne sont que les vestiges d'un aménagement plus complexe. Les alignements les plus complets montrent neuf linga au maximum alignés sur trois rangs et rien ne permet d'être certain de leur nombre d'origine, ni de la densité exacte des groupes de «Mille linga » dont les vestiges conservés se trouvent situés sur les bordures est et ouest du rocher et sur un axe médian approximativement nord-sud décalé vers la bordure ouest. Cinq cavités à fond plat, peu profondes, ont été creusées également dans la roche. Deux, situées vers la bordure sud, sont d'assez grande dimensions ; une, sur la bordure est, et deux autres, dans le prolongement de l'axe nord-sud, sont de petite taille. Elles servaient au moins dans certains cas à recevoir des linga isolés, en couple ou plus nombreux puisque ont été retrouvés dans le Mékong deux linga à tenon rectangulaire et un exemple de deux linga sculptés côte à côte sur un même socle (photo. 8). Si les deux linga isolés n'ont pu être replacés dans leur contexte originel pour la bonne raison que la taille de leur tenon ne correspond pas à la taille des mortaises creusées dans le roc, en revanche le socle du couple de linga s'intègre parfaitement dans la cavité située sur la bordure sud du rocher. Il 
est donc certain que ces cavités ont été creusées à cet effet, pour recevoir des linga rapportés. Il faut noter à ce propos que ces linga rapportés, ou du moins ceux que nous avons retrouvés, ne sont pas en grès gris comme ceux taillés dans le rocher mais en grès vert. Ce détail est d'importance car nous avions noté lors des campagnes de fouilles à Vat Phu que tous les éléments en contact avec l'eau de la source (piédestaux, conduites d'eau aériennes, etc.) étaient également sculptés dans un beau grès vert ${ }^{1}$. Cette tendance se confirme ici : un petit piédestal en bronze de style angkorien $(14,5 \times 13 \times 13 \mathrm{~cm})$, retrouvé coincé sous un pan du rocher effondré en eau profonde du côté nord, en parfaite condition et de très belle facture, sert d'écrin à un linga, d'un beau vert éclatant, peut-être en serpentine (photo. 9).

Ce rocher est donc intéressant à plusieurs titres, car, d'une part, il s'agit du quatrième exemple connu d'une transfiguration d'un fleuve ou d'une rivière en Gange par l'adjonction de reliefs illustrant le sommeil de Viṣnu et les Mille linga et, d'autre part, certains des thèmes illustrés ne se retrouvent nulle par ailleurs dans un contexte semblable. Il reste cependant à préciser l'identité de certains personnages, si c'est possible, et à réfléchir sur l'intérêt qu'avaient les Khmers à sculpter ce rocher en particulier. Doit-on, par exemple, y voir une notion de territoire et à quel monument ou site peut se rapporter ce rocher sculpté ? On sait que la rivière de Siem Reap baigne Angkor et que c'est la présence d'Angkor qui a justifié la transformation en Gange de la rivière. Qu'en est-il pour le Mékong et est-ce la présence du complexe religieux de Vat Phu qui a motivé l'ornementation de ce rocher ou de celui de Tomo ? Autant de questions auxquelles il nous faut maintenant tenter de répondre.

\section{Prospections à Mounlapamok}

En 1998, nous avons pu mener deux courtes prospections à environ $60 \mathrm{~km}$ au sud de Vat Phu, dans le district de Mounlapamok. Ce district, encore très peu peuplé, commence à peine à se développer. Il comprend de très vastes étendues de forêts et de savanes inexplolorées. Trois sites ont été visités, en compagnie de membres de la Section de la Culture de la province de Champassak ${ }^{2}$.

Ban Mai, en bordure du Mékong (10 km au nord de Mounlapamok, $5 \mathrm{~km}$ de Ban Veun Khen qui est maintenant le centre administratif du district), est sans doute un ensemble assez vaste, auquel se rattachent deux monuments, Non Pha Khao et Non Sombat, distants de $1 \mathrm{~km}$, dont ne restent que les éléments lapidaires (les monticules de briques ont été nivelés par la mise en culture et la construction de routes). On note en particulier deux linteaux du «premier style » préangkorien (makara opposés crachant un arc unique aboutissant à un médaillon central) dont l'iconographie differe toutefois. Dans un cas, les makara, montés par un petit personnage, sont accroupis et ailés et le médaillon central contient un personnage (Garuḍa?) tenant un serpent dans chaque main ${ }^{3}$. Dans l'autre, seules sont représentées les têtes des makara, un animal accroché au cou et crachant un cheval ou un cervidé, posées sur un élément architectural en forme de chapiteau ; un motif floral en rosace compose le médaillon central. Les guirlandes pendant sous l'arc ainsi que le décor intérieur de celui-ci diffèrent également.

Houay Kadian, à $26 \mathrm{~km}$ à l'ouest de Mounlapamok, est un petit temple de latérite comprenant un sanctuaire central ouvert à l'est et deux petites structures annexes au nord et au sud, contenus dans une enceinte. L'encadrement de la porte du sanctuaire est en grès. Les embrasures, de près d'un mètre de large, portent une assez longue inscription qui nous donne

1. Voir Santoni, et al., 1992.

2. Thongkhoun Boliboun, conservateur du musée de Paksé, à qui l'on doit la découverte des deux derniers sites, et Bounlap Kéokangna, conservateur des monuments de Vat Phu.

3. Ou bien Sürya? avec un lotus dans chaque main. 
pour le règne de Jayavarman Ier la plus ancienne date connue ${ }^{1}$. L'inscription paraît largement antérieure à la structure de latérite actuellement visible. Quelques fragments de statues très endommagées, probablement également postérieures (torse de Buddha, partie inférieure d'un dvārapāla accroupi) ainsi que des fragments architecturaux en grès y sont associés.

That Nang In, enfin, se trouve à plus de $46 \mathrm{~km}$ à l'ouest de Mounlapamok, à une dizaine de kilomètres de la frontière thaïlandaise, et non loin de la chaussée ancienne qui mène de Vat Phu à Angkor. Il s'agit d'un ensemble comprenant un prasat inachevé en briques, s'ouvrant à l'est, auquel un avant-corps a été rajouté (photo. 10). Deux petites structures se trouvent à l'est, de part et d'autre de l'avant-corps. L'ensemble est entouré d'une enceinte de latérite, avec gopura unique à l'est, et ceinturé de douves. Partant du gopura, une allée, flanquée de deux petites pièces d'eau, conduit à un plus vaste baray, à l'est. Enfin on peut voir au nord un autre petit baray.

La tour, de plan carré, à trois faux étages, est pourvue d'une porte à l'est et de trois fausses portes. L'encadrement de la porte, les colonnettes et le linteau sont en grès. La décoration sculptée des colonnettes octogonales est achevée, tandis que le linteau et le tympan de briques ne présentent qu'une ébauche en réserve. La cella, pillée récemment, contient un piédestal de type angkorien. L'avant-corps, de plan rectangulaire, en briques, a été plaqué postérieurement sur la façade, la masquant presque complètement. Il semble inachevé, notamment la modénature de son soubassement. L'encadrement de la porte est en grès, surmonté d'un arc de décharge en briques.

Les édicules nord-est et sud-est, très mal conservés, sont de plan rectangulaire, avec un soubassement de latérite et une élévation en briques. Ils sont ouverts à l'ouest. Quelques éléments architecturaux de grès sont visibles, épars : des éléments de colonnettes (fût, base), une fenêtre basse, rectangulaire, à petites ouvertures verticales (du type dit « fenêtre gisante »).

L'enceinte est formée d'un mur à crête soulignée à l'extérieur d'un petit ressaut. Le gopura, de plan rectangulaire, possède à l'est et à l'ouest une porte en grès, encadrée de deux colonnettes à fût lisse (inachevé ?). Les superstructures de briques en encorbellement comportent deux arcs de décharge.

À première vue, les différentes structures n'appartiennent manifestement pas à la même époque. On notera que la modénature du prasat est particulièrement fine et soignée. Mais l'état d'inachèvement ne permet toutefois pas d'attribuer de date précise.

Un autre temple, That Ban Don, que nous n'avons pu visiter, nous a été signalé. Il s'agirait d'une tour de briques à décor de pilastres. Toute la région au sud de Vat Phu, en particulier à partir de Ban That (trois chapelles du XIIe siècle) par où passe la chaussée ancienne, mérite une exploration systématique. Il serait notamment intéressant de pouvoir suivre tout du long cette chaussée ancienne qui, d'après l'examen des photographies aériennes, semble ponctuée de grands baray et de structures monumentales. Cette exploration fera l'objet, si les circonstances le permettent, d'une prochaine campagne.

Marielle SANTONI (CNRS, UMR 9993) et Christine HAWIXBROCK (EFEO)

1. Claude Jacques nous communique les informations suivantes : l'inscription (encore en cours de déchiffrement) a été répertoriée sous le $n^{\circ} \mathrm{K} 1201$; partie en sanskrit, partie en khmer, elle mentionne le nom de Jayavarman, le nom du personnage célébré, son titre, son lieu d'habitation (Śrī Kuruksetra), la date précise (dimanche 18 mai 654 [calendrier julien], à 5 h40 du matin [temps civil local]), l'objet de sa fondation (un ștāneśvara, le « Seigneur impassible », probablement un linga), les donations (rizières, serviteurs, haches), des listes d'esclaves. On notera que Kurukșetra est le nom du tirtha qui serait la ville ancienne de Vat Phu, fondé au milieu du $V^{e}$ siècle par le roi Devānīka (stèle de Vat Luang Kau, voir l'introduction de cette chronique). Il s'agit de la troisième occurrence de ce nom dans toute l'épigraphie khmère. 


\section{RÉFÉRENCES BIBLIOGRAPHIQUES}

CEDÈs, G.

1956 « Nouvelles données sur les origines du royaume khmèr. La stèle de Vat Luong Kau, près de Vat P'hu », BEFEO, 48 (1) p. 209 - 220.

1989 Les états hindouisés d'Indochine et d'Indonésie, Paris, (réimpression de l'édition de 1964), De Boccard.

CUCARZI, M., ZOLESE, P.

1994 «An attempt to inventory khmer monumental remains through geomagnetic modelling: the ancient city of Vat Phou », in P.-Y. Manguin éd., Southeast Asian Archaeology 1994,

HAWIXBROCK, C. Centre For South-East Asian Studies, University of Hull.

1998 «From the Mekong to the Ganga, a carved rock near Vat Phu », communication présentée à la 7 th International Conference of the European Association of Southeast Asian Archaeologists, Berlin, 31 août- 4 septembre 1998.

JACQUES, C.

1962 « Notes sur l'inscription de la stèle de Vat Luong Kau », Journal asiatique, p. 249 - 256.

1986 «Le pays khmer avant Angkor», Journal des Savants, janvier-septembre 1986, p. 59-95.

1993 «Mahendravarman's campaigns in North-East Thailand : new evidence from the inscriptions of Wat Phu », Paper presented at the Symposium on Thai Studies, London, July 1993.

PARMENTIER, $\mathrm{H}$.

1914 «Le temple de Vat Phu», BEFEO, 14 (2), p. 1 - 31.

1927 L'art khmèr primitif, Paris, PEFEO, 22, 2 vol.

1933 «L'art présumé du Fou-Nan », BEFEO, 32, p. 183-189.

PELLIOT, P.

1903 «Le Fou-nan », BEFEO, 3, p. 303 - 348.

SANTONI, M. et al.

1991 «Approche archéologique de l'histoire du Laos : l'exemple de Vat Phou », Second Symposium Franco-Thaï : Récentes recherches en archéologie en Thaïlande. Bangkok, Silapakorn University, p. 194 - 215

SANTONI, M. et al.

1998 «Excavations at Champassak and Wat Phu, South Laos. » in R. Ciarla ed., Southeast Asian Archaeology 1992, Rome, ISIAO.

SANTONI, M.

1993 «Résultat des fouilles 1993 d'un monument de la ville ancienne associée à Vat Phu (Champassak, Laos)», in Cl. Jacques éd., Symposium : Sources de l'Histoire du Pays Khmer, Paris 28 juin - 2 juillet 1993, sous presse.

1994 «Fouille d'un monument préangkorien dans la ville ancienne associée à Vat Phou (province de Champassak, Laos) », in P.-Y. Manguin éd., Southeast Asian Archaeology 1994, Centre For South-East Asian Studies, University of Hull (sous presse).

1996 « Results of recent excavations in Champassak, southern Laos : season 1995 -1996 », Communication présentée à la 6th International Conference of the European Association of Southeast Asian Archaeologists, Leyden, 2 - 6 septembre 1996.

1998 «Results of 1998 fieldwork in Vat Phu area (Champassak Province, Laos) », communication présentée à la 7 th International Conference of the European Association of Southeast Asian Archaeologists, Berlin, 31 août- 4 septembre 1998. 
SOUKSAVATDY, V.

1994 "Site de production de briques dans la ville ancienne près de Vat Phou (province de Champassak, Laos) », in P.-Y. Manguin, éd., Southeast Asian Archaeology 1994, Centre for South-East Asian Studies, University of Hull.

1996 «Excavation of a pre-Angkorian monument in the Old City of Wat Phu (Champassak Province, Laos) », Communication présentée à la 6th International Conference of the European Association of Southeast Asian Archaeologists, Leyden, 2-6 septembre 1996.

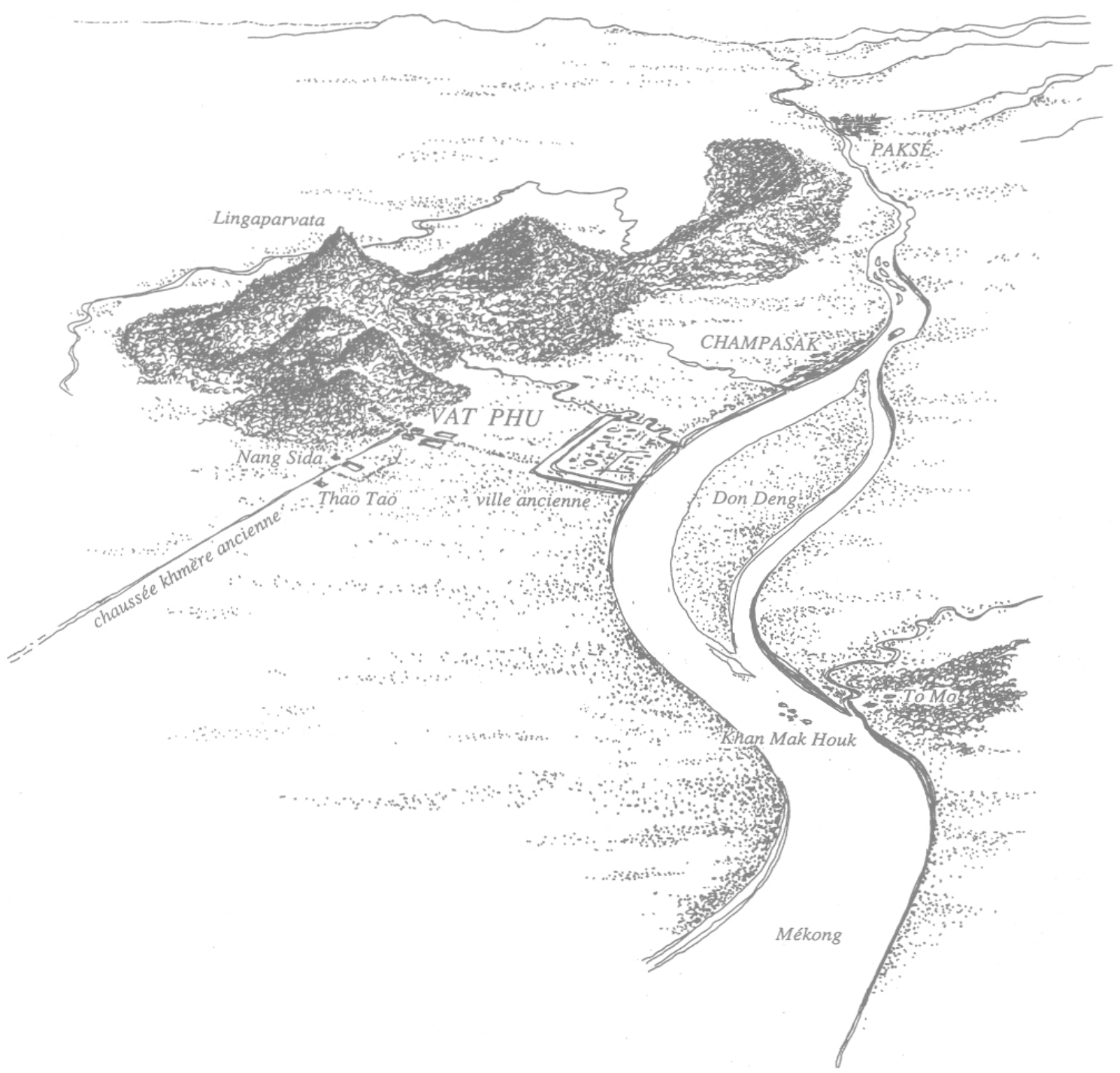

Fig. 1 : zone archéologique de Vat Phu, vue du sud (dessin, (C Pierre Pichard). 


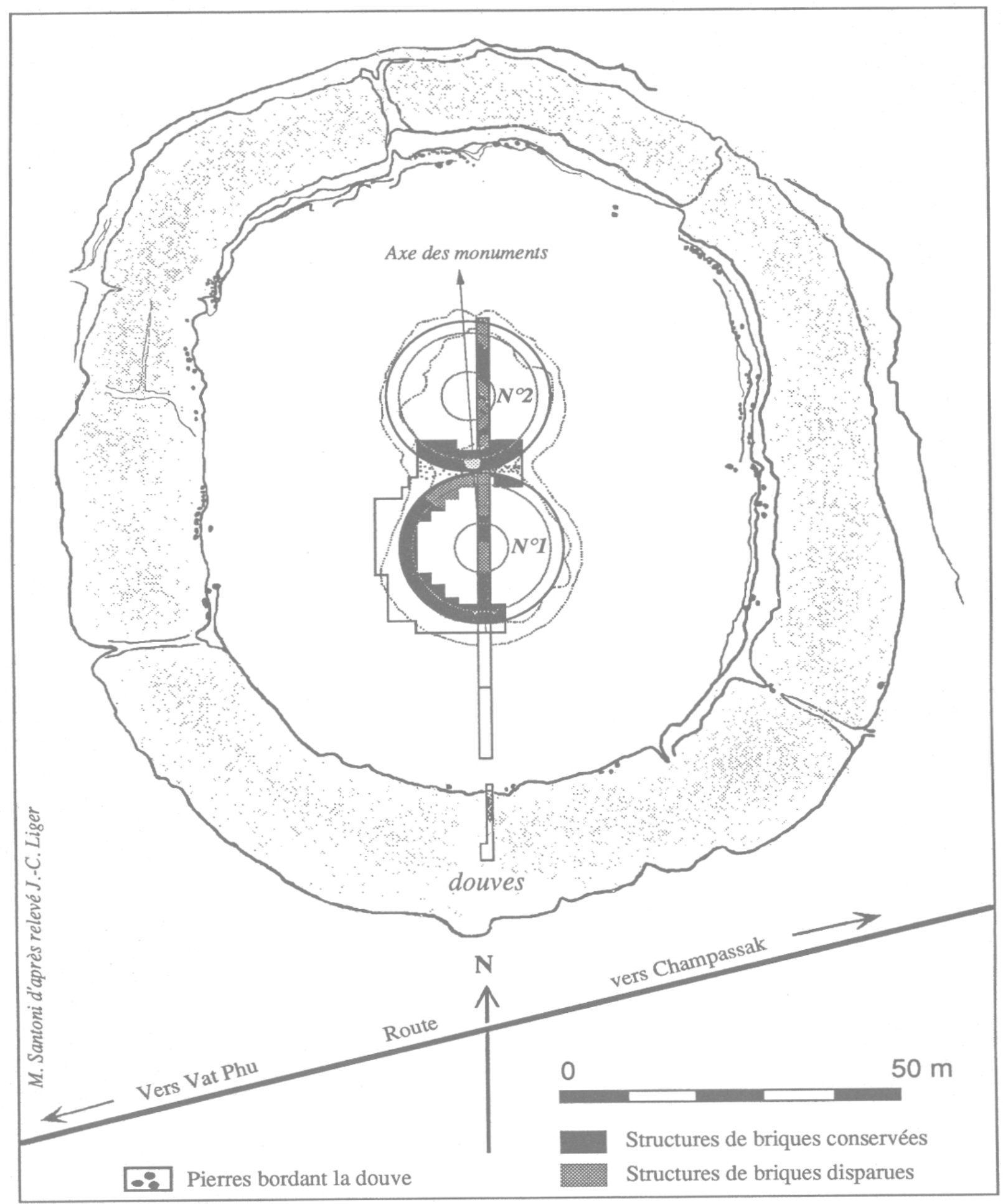

Fig. 2 : plan du site de Nong Vienne. 


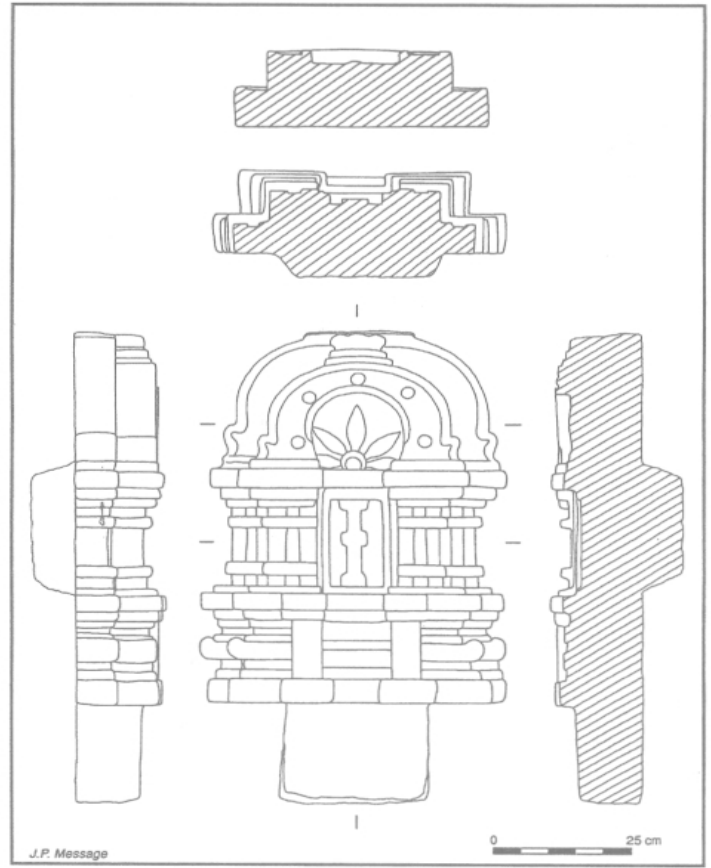

Fig. 3 :

Panneau décoratif de grès, Nong Vienne.

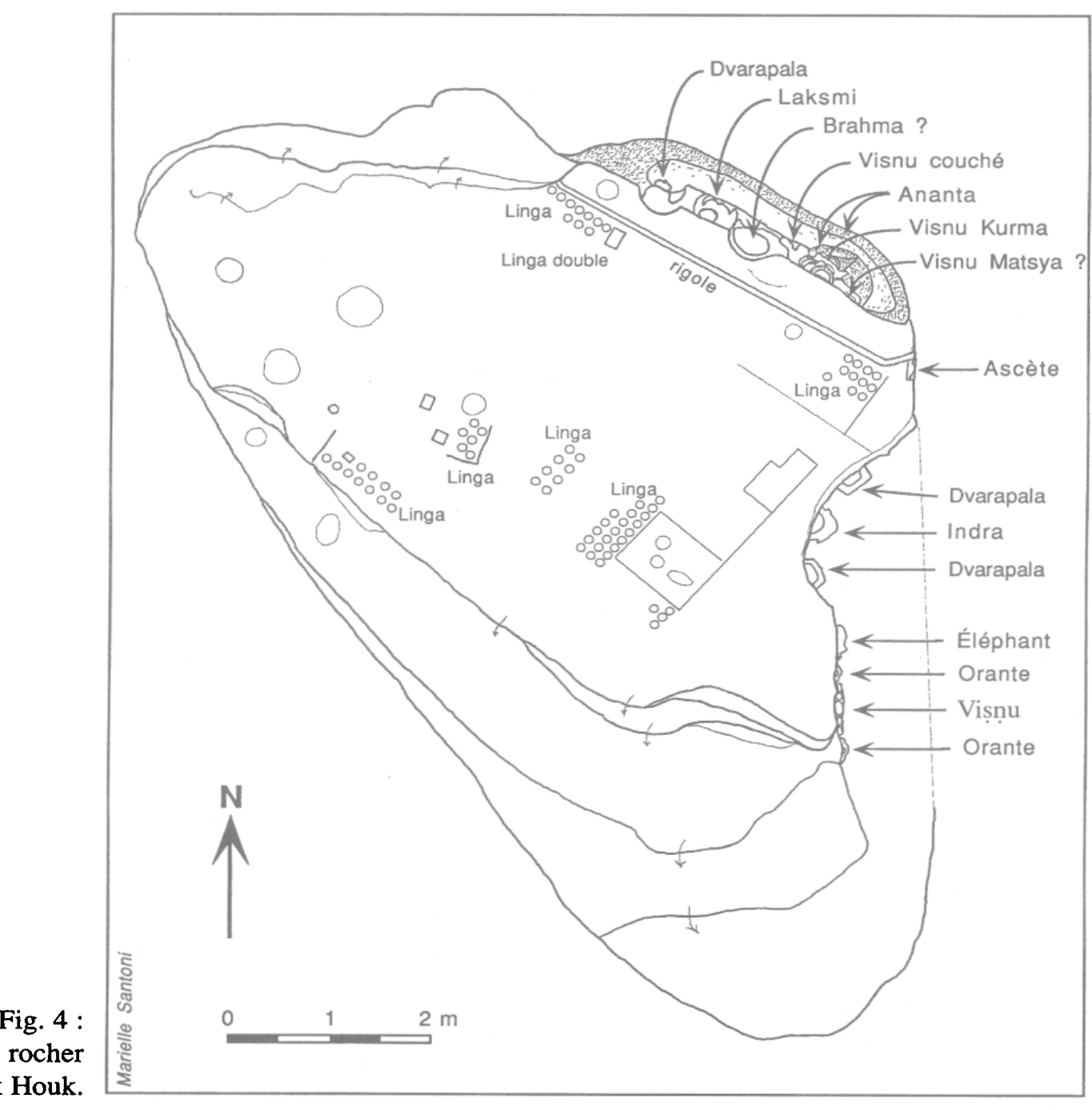

plan du rocher

de Khan Mak Houk. 


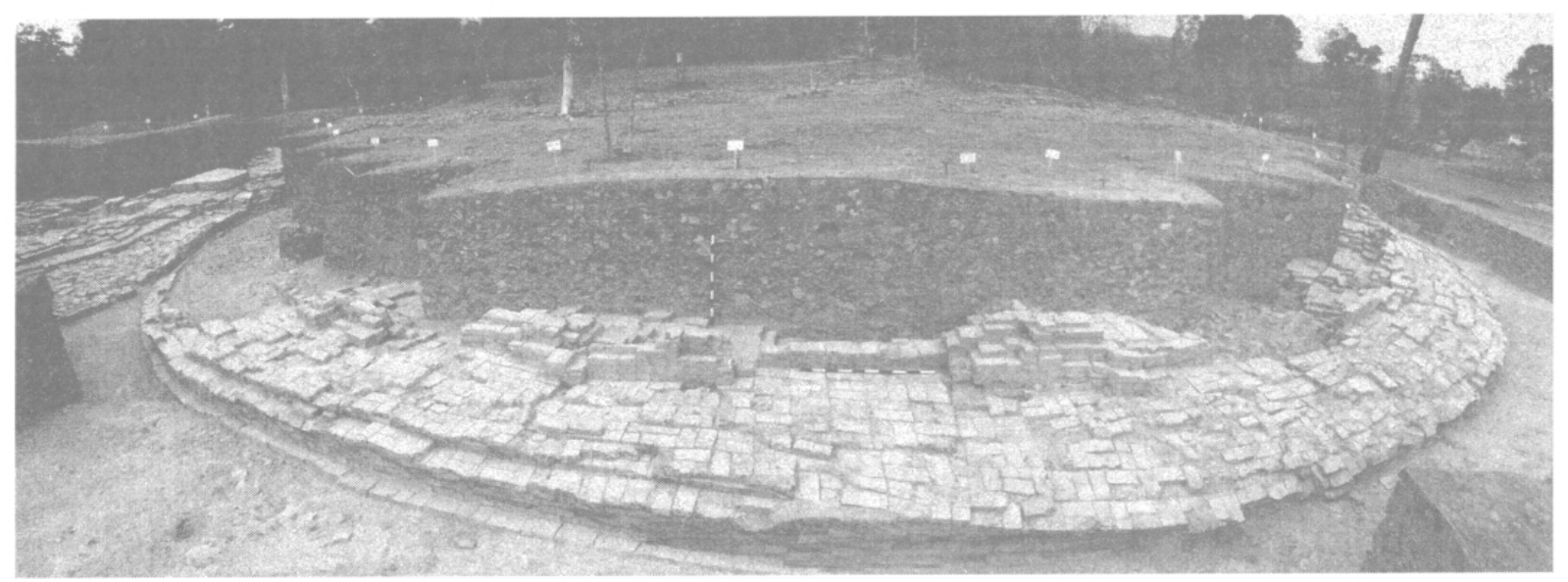

Photo. 1 : Nong Vienne, le monument sud $\left(\mathrm{n}^{\circ} 1\right)$ vu de l'ouest. (Les photographies sont de Marielle Santoni).

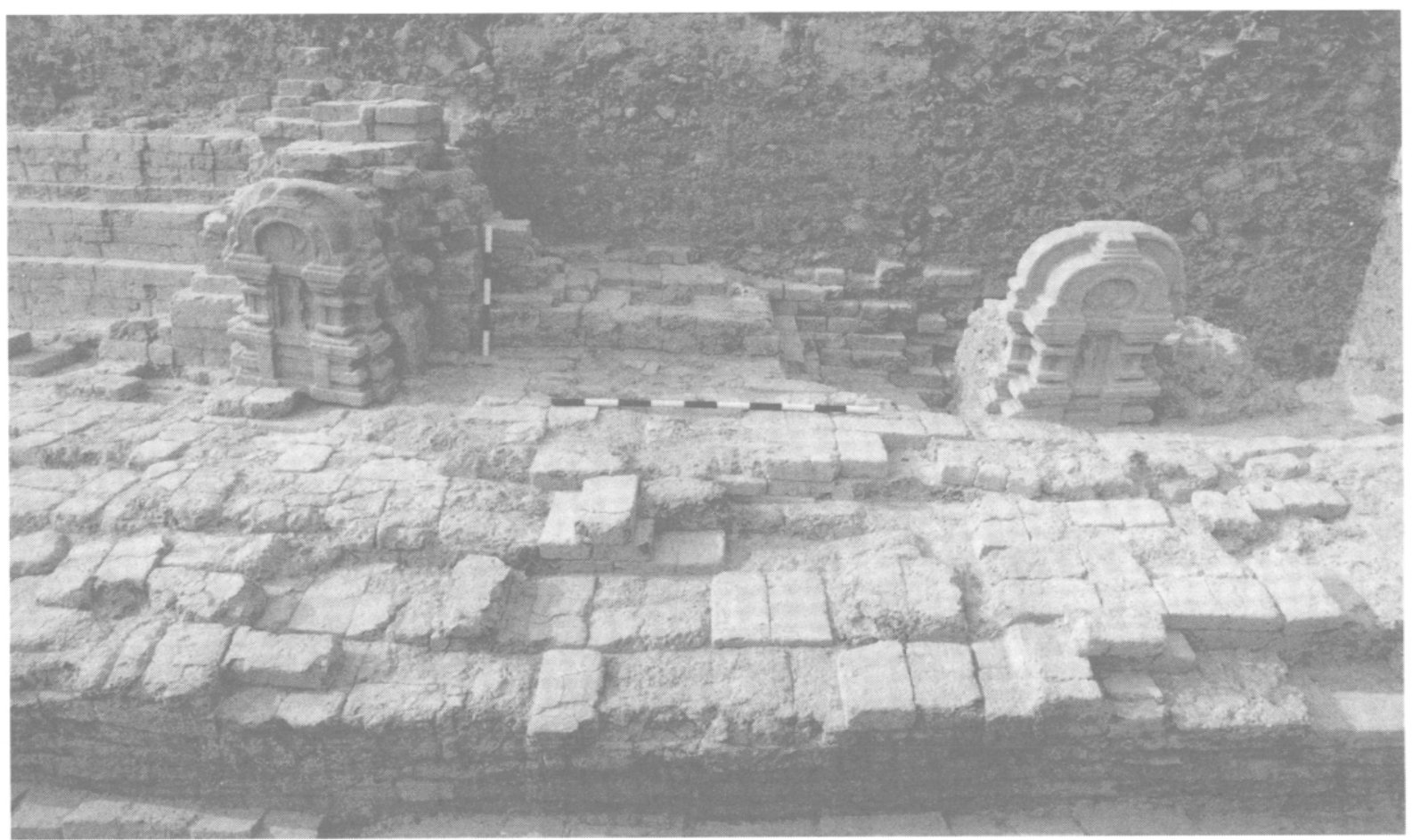

Photo. 2 : Nong Vienne, entrée sud du monument sud $\left(n^{\circ} 1\right)$ 

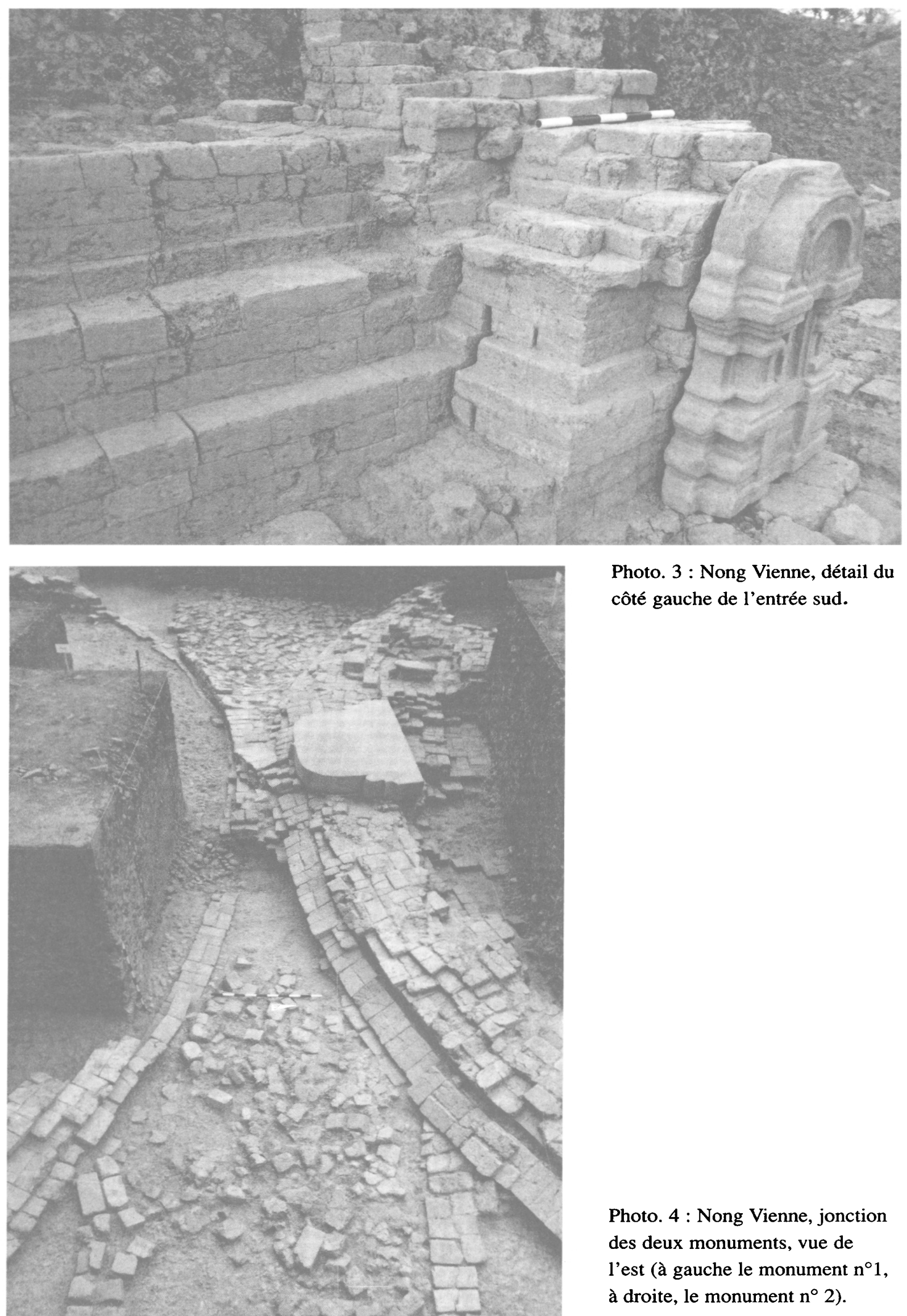

Photo. 3 : Nong Vienne, détail du côté gauche de l'entrée sud.

Photo. 4 : Nong Vienne, jonction des deux monuments, vue de l'est (à gauche le monument $n^{\circ} 1$, à droite, le monument $n^{\circ} 2$ ). 


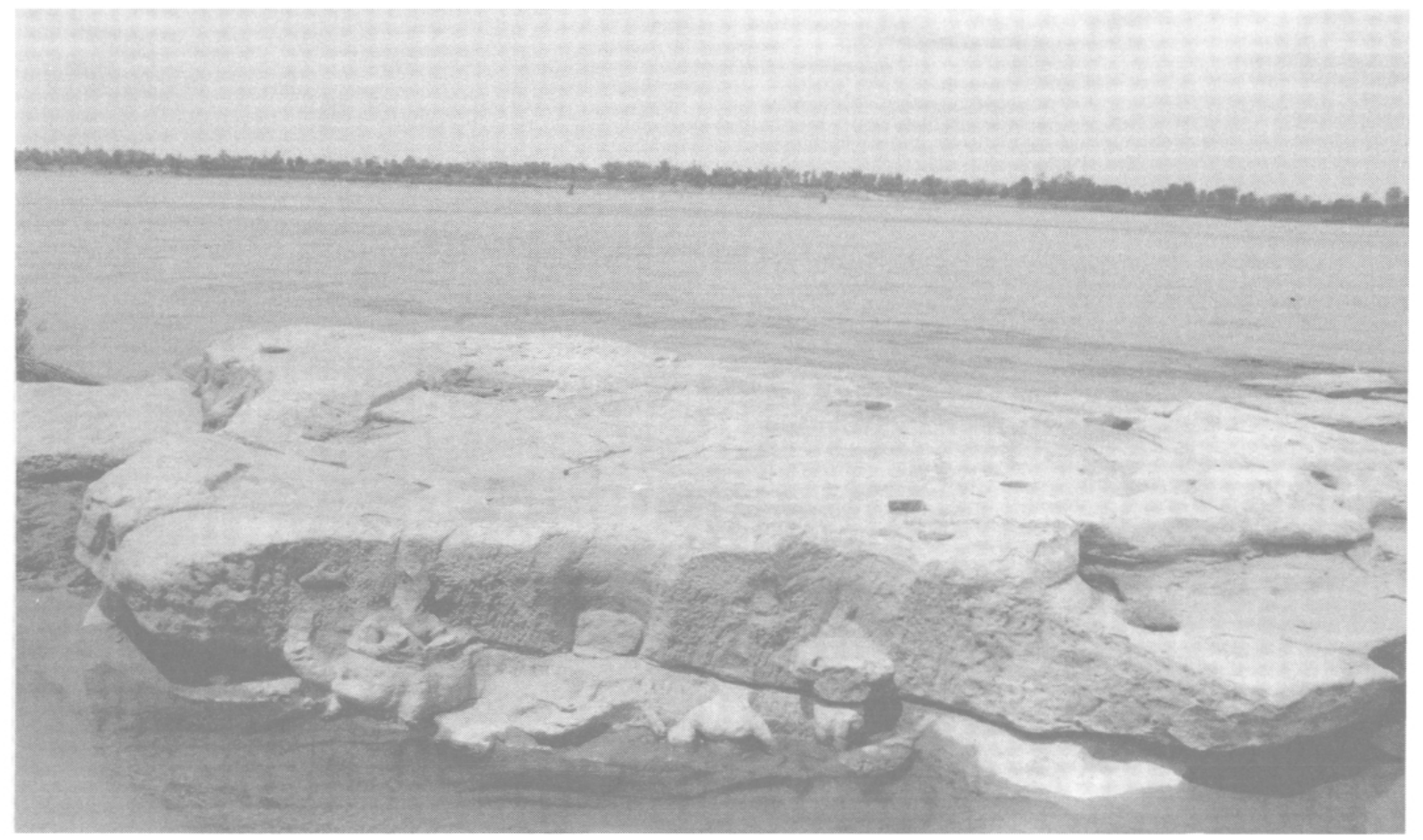

Photo. 5 : Khan Mak Houk, vue de la face nord-est : Viṣnu couché.

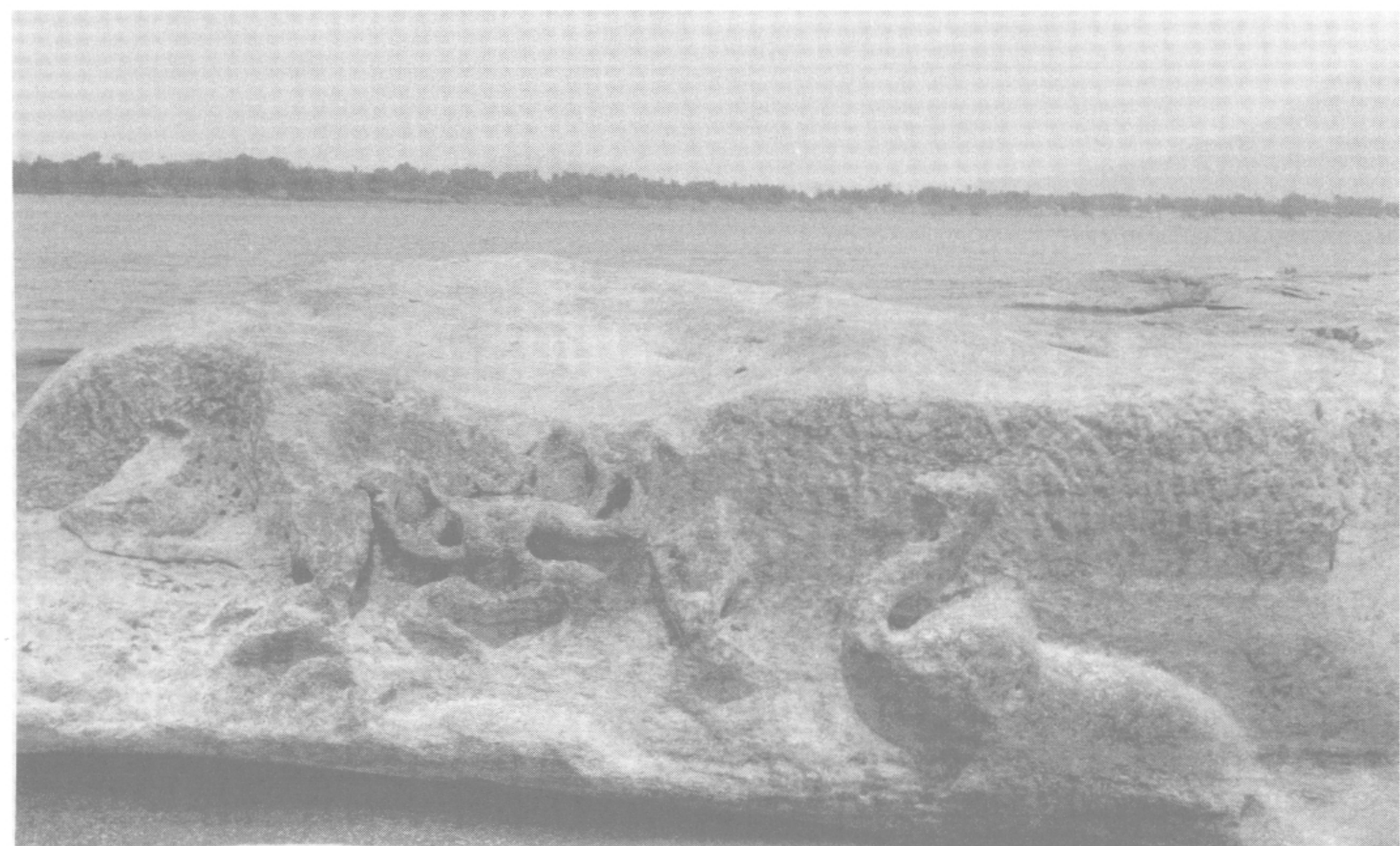

Photo. 6 : Khan Mak Houk, vue de la face est (partie sud): ondoiement de Lakșmī. 


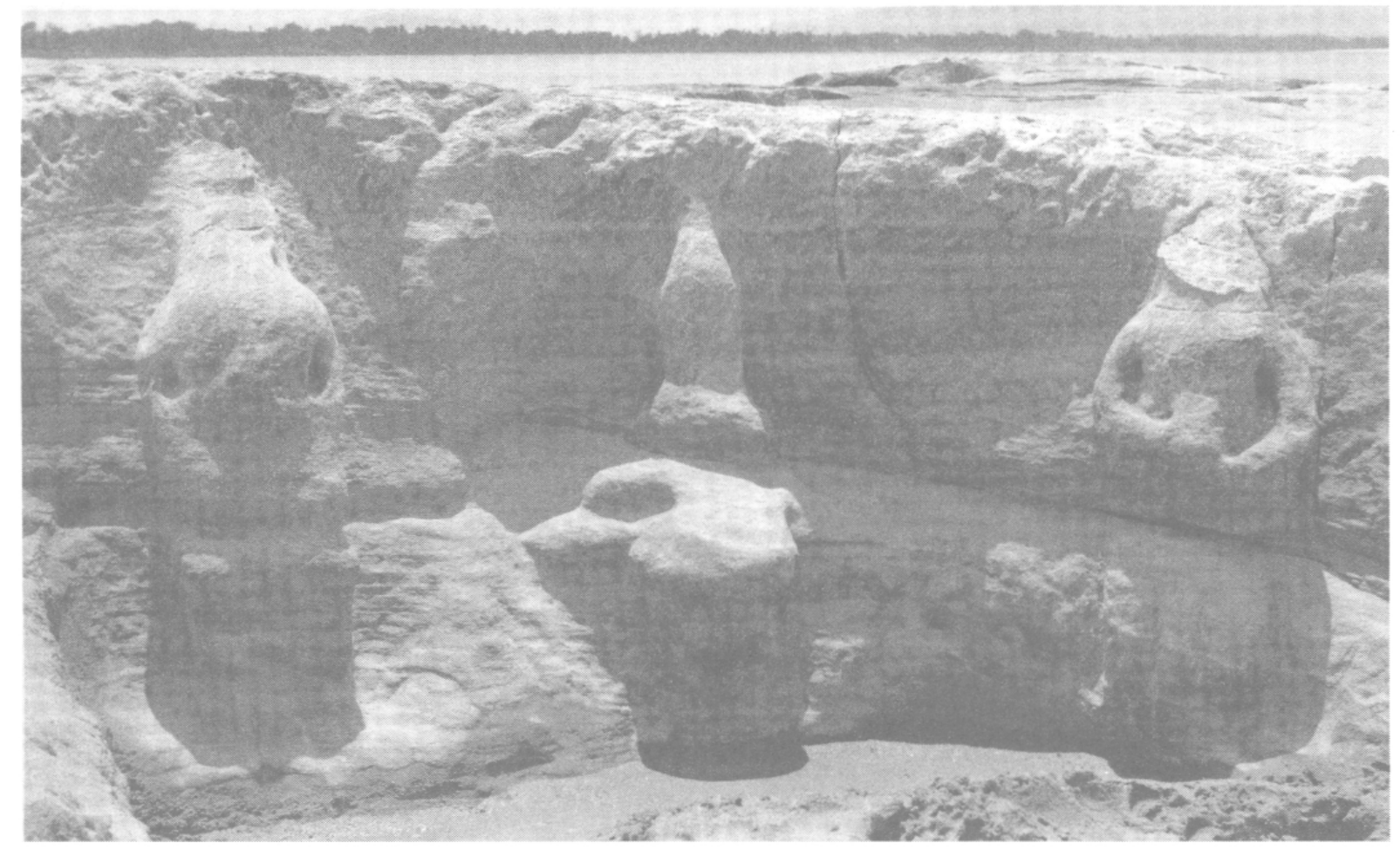

Photo. 7 : Khan Mak Houk, vue de la face est (partie nord) : Indra (?) entre deux dvārapāla.

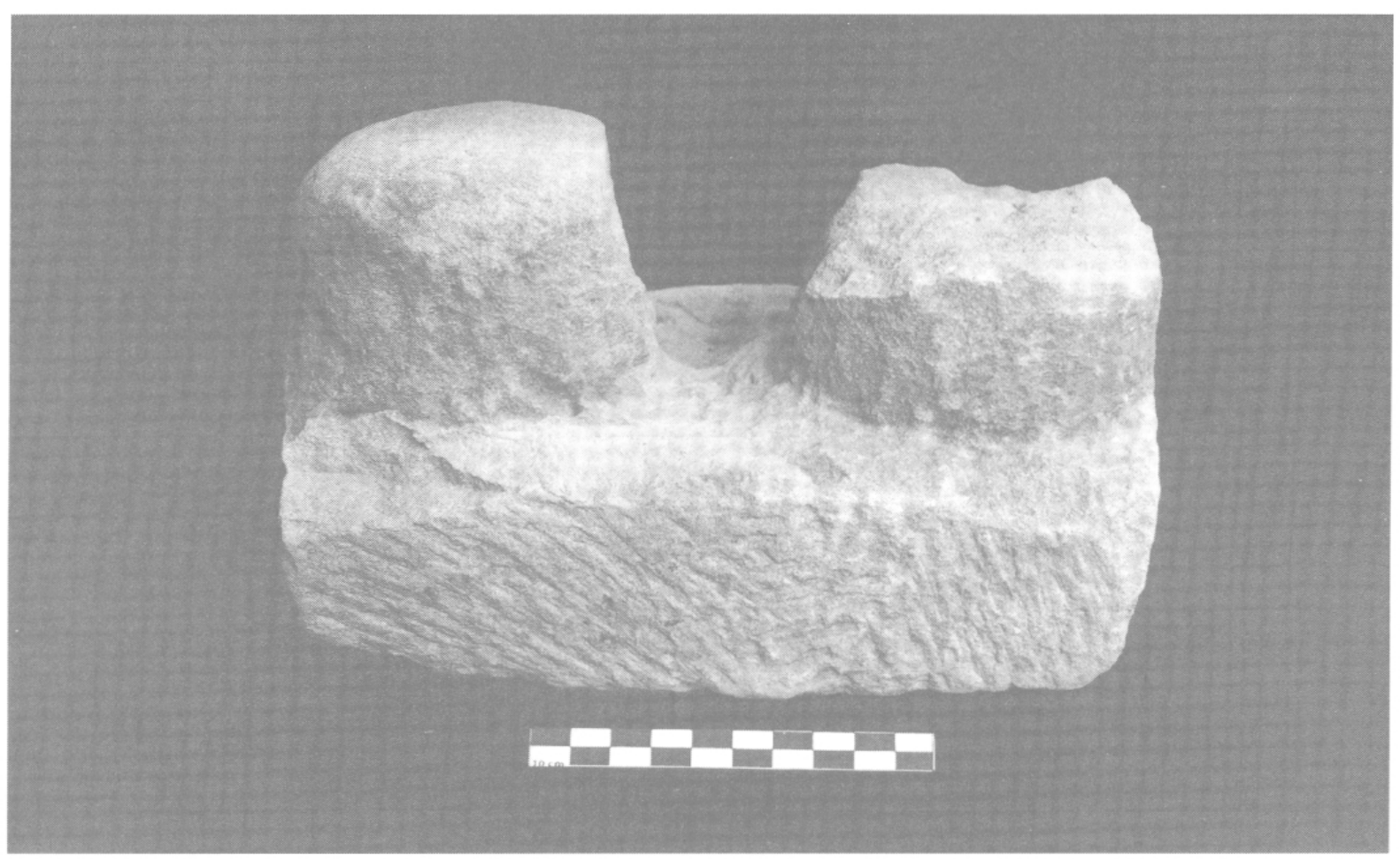

Photo. 8 : Khan Mak Houk, deux linga de grès vert sur socle. 


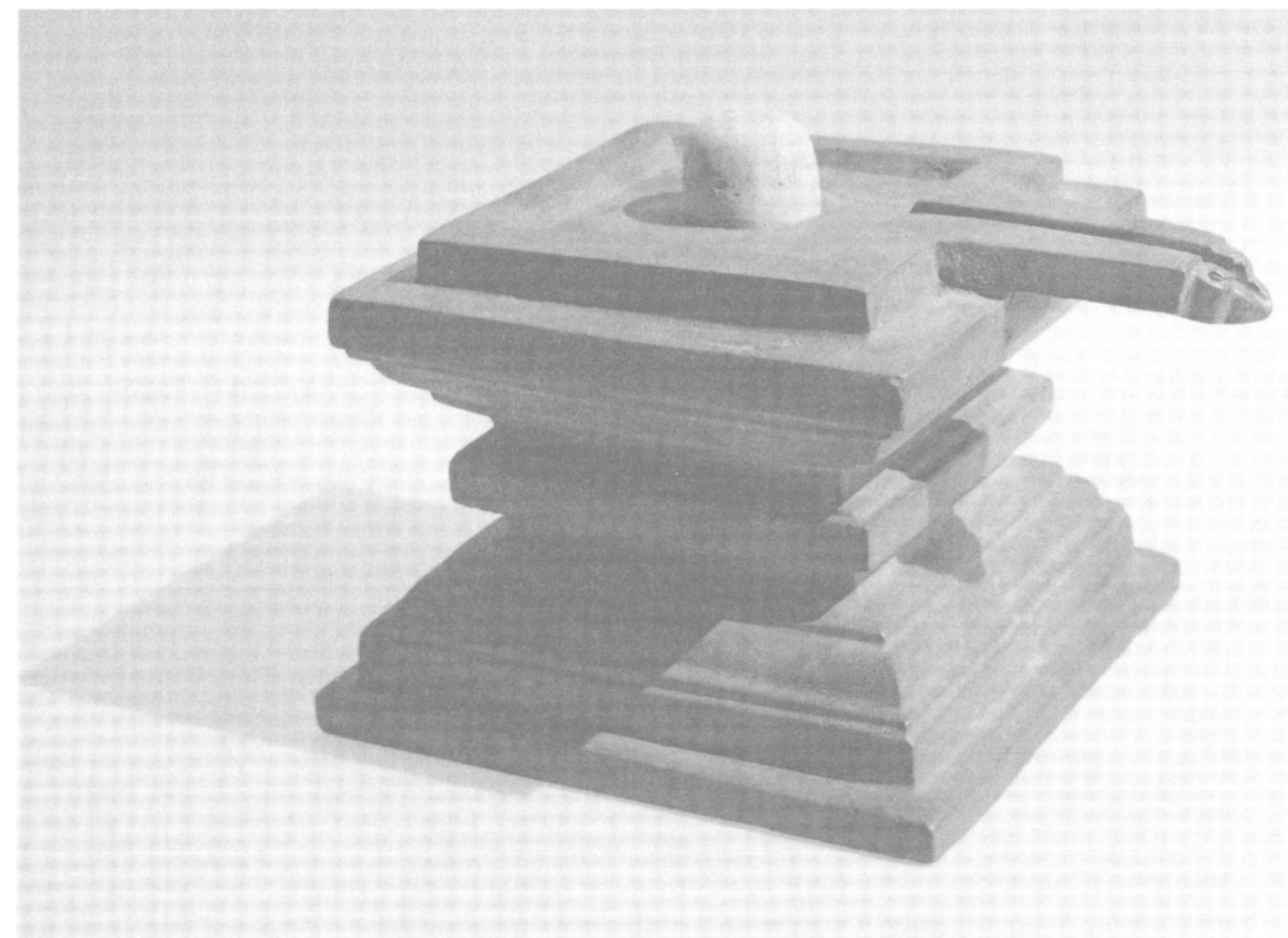

Photo. 9 : Khan Mak Houk, piédestal de bronze avec linga de pierre verte.

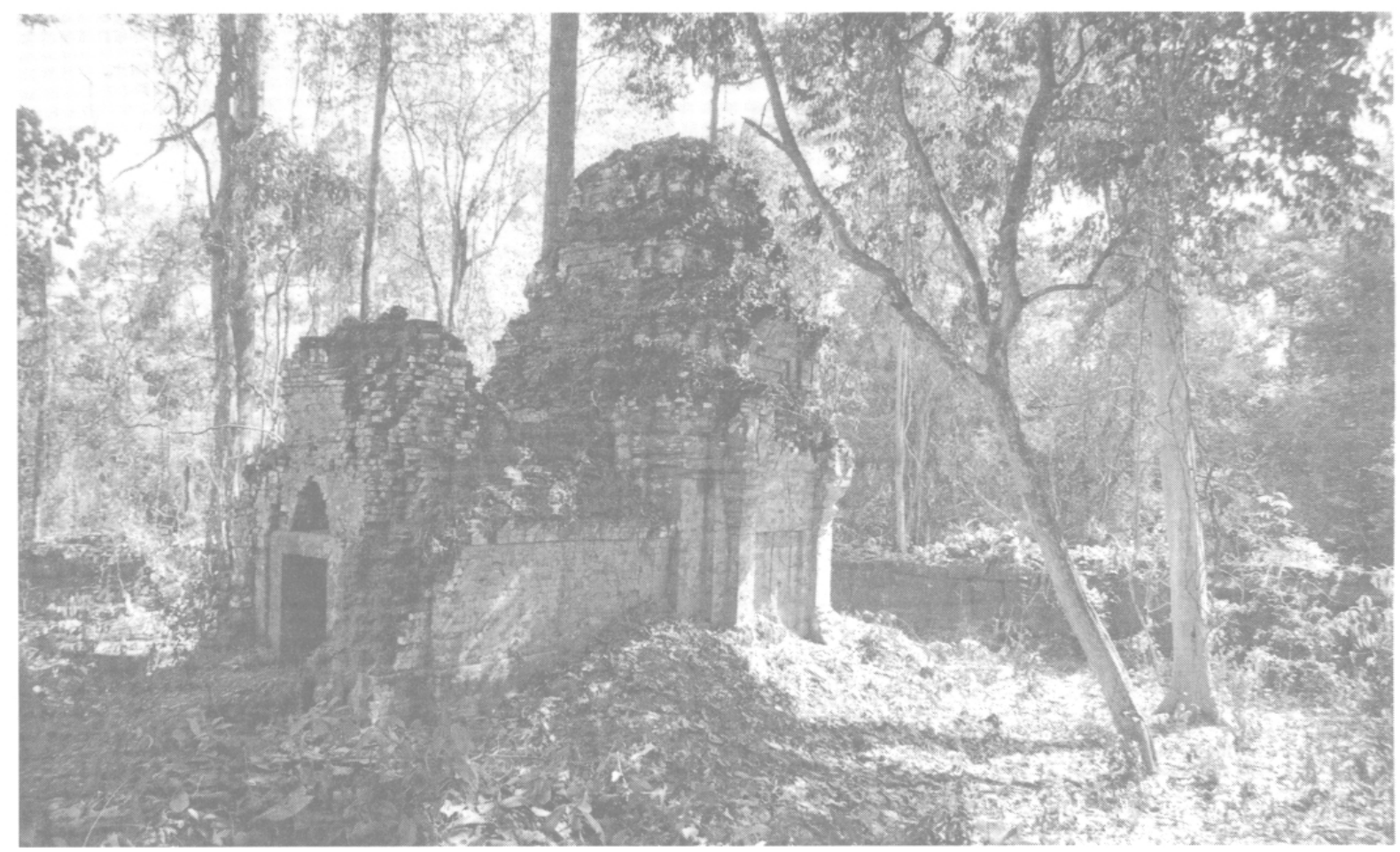

Photo. 10 : That Nang In, le prasat et son avant-corps, vus du nord-est. 